is self-limiting. Most patients do not require treatment with steroids [1].

IgG4-related disease is a relatively newly discovered entity [4, 5] It is characterised by an inflammatory and fibrosing infiltrate rich in IgG4-positive plasma cells and often elevated IgG4 serum concentrations. The most well-known affected organ is the pancreas, a disease formerly known as auto-immune pancreatitis. However, all internal organs may be affected, for instance the salivary glands (Sjögren-like syndrome), retroperitoneum (retroperitoneal fibrosis), kidney (interstitial nephritis), lymph nodes and the aorta (inflammatory aneurysm) [4]. The aetiology and pathogenesis are not known. Early IgG4-related disease is very steroid responsive with quick and longstanding remissions, and decreases in serum IgG4 levels. In cases with extensive fibrotic lesions, remission after therapy is less likely.

IgG4-related disease is also known for its pulmonary involvement. Nodular, bronchovascular, pleural, alveolar interstitial and round-shaped ground-glass opacity presentations have been described previously [6-8]. All presentations showed an infiltration of IgG4 positive plasma cells with sclerosing inflammation in the respective tissues, with concomitant increased plasma levels of IgG4. Pulmonary involvement frequently coincides with extrapulmonary disease, such as pancreas, retroperitoneum and kidney. In most cases, steroid therapy is effective.

Hypergammaglobulinaemia has long been considered part of Rosai-Dorfman disease. Rosai-Dorfman disease was only recently associated with increased $\operatorname{IgG} 4$ cell populations $[9,10]$. This suggests a possible overlap or even a common cause between Rosai-Dorfman disease and IgG4-related disease. One could even speculate that Rosai-Dorfman disease might be a reactive pattern to IgG4-related disease. The underlying pathophysiology of this overlap and the clinical significance with respect to treatment and prognosis is not yet clear.

In summary, we present a patient who was admitted for suspected lung cancer but who was eventually diagnosed with two rare disease patterns: Rosai-Dorfman disease and IgG4related disease. IgG4-related disease is a relatively underdiagnosed condition in which increasing scientific interest exists. There is evidence of an overlap between these entities (Rosai-Dorfman disease might be a reactive pattern to IgG4related disease), but the cause and implications of this overlap are not known.
Wouter K. de Jong*, Philip M. Kluin" and Harry J.M. Groen* *Dept of Pulmonary Diseases, University Medical Center Groningen, and "Dept of Pathology and Medical Biology, University Medical Center Groningen, Groningen, The Netherlands.

Correspondence: W.K. de Jong, Dept of Pulmonary Diseases, University Medical Center Groningen, P.O. Box 30.001, 9700 RB Groningen, the Netherlands. E-mail: w.k.de.jong@umcg.nl

Statement of Interest: None declared.

Provenance: Submitted article, peer reviewed.

\section{REFERENCES}

1 Pulsoni A, Anghel G, Falcucci P, et al. Treatment of sinus histiocytosis with massive lymphadenopathy (Rosai-Dorfman disease): report of a case and literature review. Am J Hematol 2002; 69: 67-71.

2 Foucar E, Rosai J, Dorfman R. Sinus histiocytosis with massive lymphadenopathy (Rosai-Dorfman disease): review of the entity. Semin Diagn Pathol 1990; 7: 19.

3 Ali A, Mackay D. Rosai-Dorfman disease of the lung. Thorax 2009; 64: 908-909.

4 Bateman AC, Deheragoda MG. IgG4-related systemic sclerosing disease - an emerging and under-diagnosed condition. Histopathology 2009; 55: 373-383.

5 Stone JH, Zen Y, Deshpande V. IgG4-related disease. N Engl J Med 2012; 366: 539-551.

6 Zen Y, Inoue D, Kitao A, et al. IgG4-related lung and pleural disease: a clinicopathologic study of 21 cases. Am J Surg Pathol 2009; 33: 1886-1893.

7 Shigemitsu H, Koss MN. IgG4-related interstitial lung disease: a new and evolving concept. Curr Opin Pulm Med 2009; 15: 513-516.

8 Ryu JH, Sekiguchi H, Yi ES. Pulmonary manifestations of immunoglobulin G4-related sclerosing disease. Eur Respir J 2012; 39: $180-186$.

9 Roberts SS, Attanoos RL. IgG4+ Rosai-Dorfman disease of the lung. Histopathology 2010; 56: 662-664.

10 Shrestha B, Sekiguchi H, Colby TV, et al. Distinctive pulmonary histopathology with increased IgG4-positive plasma cells in patients with autoimmune pancreatitis: report of 6 and 12 cases with similar histopathology. Am J Surg Pathol 2009; 33: 1450-1462.

DOI: $10.1183 / 09059180.00001612$

\title{
Bronchial rupture related to endobronchial stenting in relapsing polychondritis
}

\section{To the Editor:}

Relapsing polychondritis is a rare multi-systemic disease characterised by recurrent episodes of inflammation and destruction of cartilaginous structures [1]. Airway involvement by relapsing polychondritis, which results in tracheobronchomalacia and airway stenosis, is associated with a poor prognosis.
Several reports have suggested that endobronchial intervention can be beneficial in these subjects $[2,3]$. Herein, we describe a case of bronchial rupture related to endobronchial intervention in a patient with relapsing polychondritis.

A 47-yr-old male nonsmoker was referred for management of progressive dyspnoea, stridor and cough evolving over 
12 months. He had been considered to have severe asthma and was treated with a fixed combination of inhaled corticosteroids and long-acting $\beta$-agonists, and short courses of oral corticosteroids. Spirometry showed fixed airflow limitation with a postbronchodilator forced expiratory volume in $1 \mathrm{~s}$ (FEV1)/forced vital capacity of $22 \%$ and an FEV1 of $22 \%$ predicted. A thoracic computed tomography (CT) scan revealed marked thickening and narrowing of trachea and mainstem bronchi (fig. 1). A diagnosis of relapsing polychondritis was suggested; the patient had no auricular or nasal chondritis and no ocular symptoms. Fluorodeoxyglucose positron emission tomography showed no cartilaginous tracer uptake. Bronchoscopy showed marked thickening and inflammation of airway mucosa. Severe tracheobronchomalacia, which was observed predominantly in the left mainstem bronchus, further contributed to airway stenosis with marked reduction in airway calibre. Careful balloon dilatation of the left mainstem bronchus was performed but was insufficient to restore airway patency. An attempt to deploy a bifurcated silicone stent (Tracheobronxane ${ }^{\circledR}$ Dumon $^{\mathrm{TM}}$; Novatech, La Ciotat, France) in the trachea and main bronchi was unsuccessful due to incomplete stenosis of the left mainstem bronchus, and the stent had to be removed immediately. After this intervention the patient experienced isolated subcutaneous emphysema without bronchoscopic visualisation of bronchial rupture and without radiographic pneumothorax. 3 days later, the subcutaneous emphysema worsened rapidly and acute respiratory failure required emergency invasive mechanical ventilation. Bronchoscopy revealed laceration of the mainstem left bronchus, leading to a decision for surgical bronchial repair. The patient's haemodynamic condition deteriorated rapidly due to a left tension pneumothorax and pneumopericardium, which required pericardium decompression and insertion of a left chest tube. Surgical bronchial repair was impossible due to extensive laceration and a left pneumonectomy was performed. Histological examination of the bronchi revealed pleomorphic inflammatory infiltrate with disrupted cartilage structures, compatible with the diagnosis of relapsing polychondritis (fig. 2). The post-operative period was complicated by prolonged mechanical ventilation with several episodes of ventilatorassociated pneumonia. Weaning from mechanical ventilation was finally achieved after 6 weeks without a tracheostomy. Treatment with methotrexate ( $25 \mathrm{mg}$ per week) and prednisone (7 mg per day) was started. Within a few weeks respiratory symptoms improved dramatically. A bronchoscopy showed improvement in airway patency and the patient was discharged from hospital.

Relapsing polychondritis is a rare disease and therapies are based on clinical experience rather than on results from clinical trials [1]. Immunosuppressive therapies, including corticosteroids, methotrexate, cyclophosphamide and tumour necrosis factor- $\alpha$ blockers, have been proposed for subjects with relapsing polychondritis [1]. ENG and SABANATHAN [2] further proposed that bronchoscopic intervention could be useful when tracheobronchial stenosis and/or malacia occurred in subjects with relapsing polychondritis. Although immunosuppressive therapy and bronchoscopic intervention were often associated, no study has addressed the best treatment strategy in subjects with airway involvement and relapsing polychondritis.

In our report, an attempt to perform balloon dilatation with endobronchial stenting resulted in bronchial laceration and rupture, leading to left pneumonectomy. ERNST et al. [3] have described the largest cohort of subjects with relapsing polychondritis and airway involvement $(n=31)$. Those authors performed bronchoscopy in 23 subjects and 12 patients underwent bronchoscopic interventions (stenting and/or balloon dilatation). No major complications were described and most patients experienced improvement in airway symptoms [3]. Interestingly, Dooms et al. [4] conducted a retrospective study of the clinical effectiveness of self-expandable metallic stents in 17 patients with benign airway strictures. Tracheal tear was the single immediate complication of stenting, and occurred in a
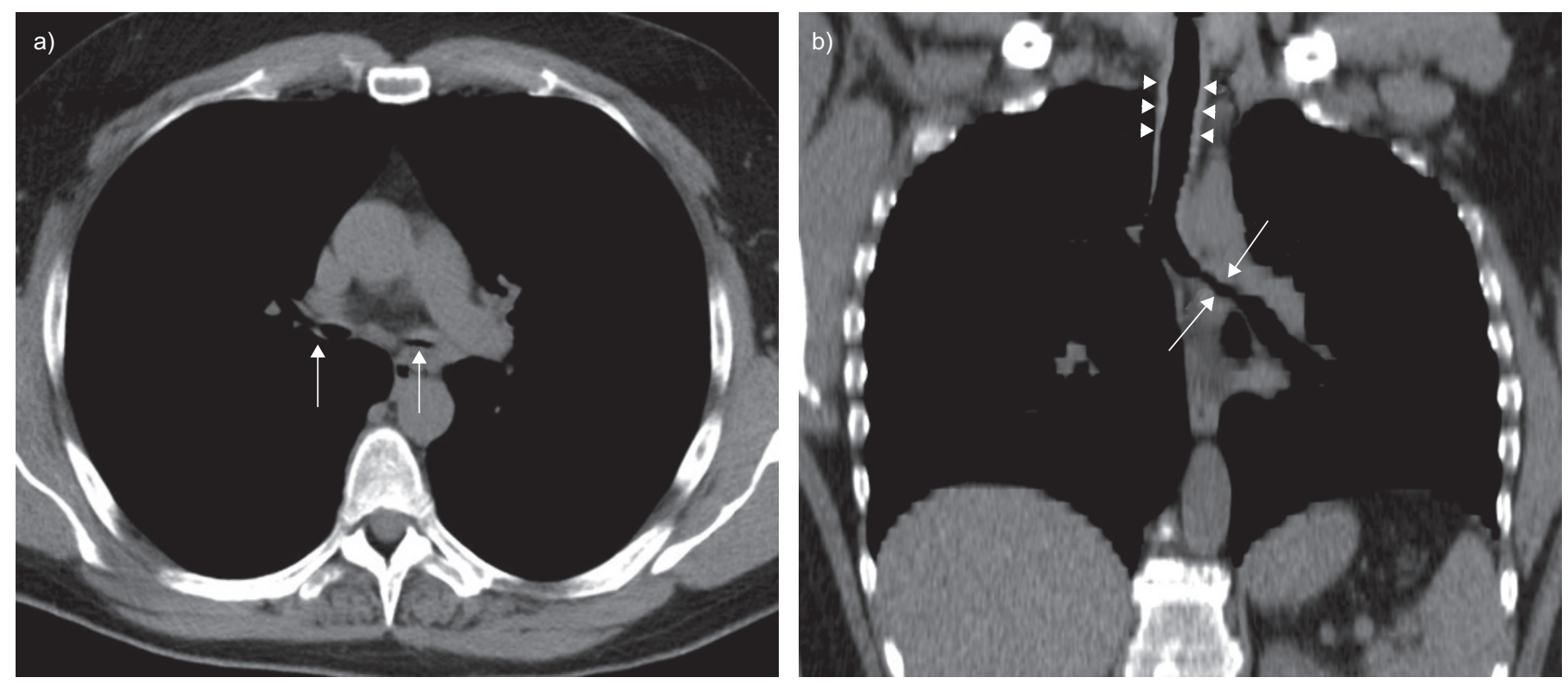

FIGURE 1. a) An inspiratory transversal slice thoracic computed tomography (CT) scan revealing marked narrowing of mainstem bronchi (arrows). b) A coronal slice CT scan indicating tracheal wall thickening (arrowheads) and severe narrowing of the left mainstem bronchus (arrows). 


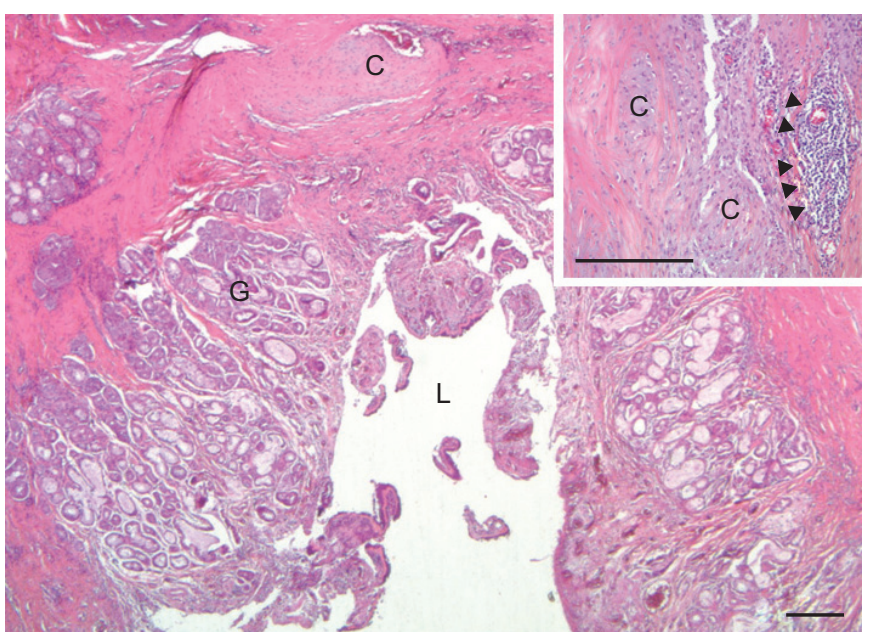

FIGURE 2. Representative photomicrographs of structural abnormalities in the left mainstem bronchus (surgical resection). Sections were stained with haematoxylin and eosin. The airway wall contains hypertrophic submucosal glands (G) surrounded by damaged cartilage $(C)$. The airway lumen $(L)$ is markedly narrowed. At higher magnification (inset), inflammatory cells (arrowheads) infiltrate cartilaginous structures. Scale bar $=200 \mu \mathrm{m}$; inset scale bar $=50 \mu \mathrm{m}$.

subject with relapsing polychondritis [4]. Although bronchoscopic intervention could be useful in subjects with airway involvement and relapsing polychondritis, the risk of serious complications related to laceration of the fragile inflammatory bronchi is probably increased compared to other causes of benign stenosis.

Multiple mechanisms may account for airway narrowing in patients with relapsing polychondritis [3]. Tracheal/tracheobronchial wall thickening, which is related to the inflammatory process, could result in airway stenosis and fibrosis. Cartilage destruction, which is presumably associated with decreased airway wall thickness, is responsible for tracheobronchomalacia characterised by airway collapse on active exhalation [3]. Detailed assessment of the characteristics of the airway wall using a combination of CT scan, bronchoscopy and/or endobronchial ultrasound is probably important in identifying the mechanisms of airway narrowing. It is suggested that endobronchial intervention is more appropriate for inflammatory/fibrous narrowing with increased wall thickness and more dangerous in cases of dominant malacia with reduced airway wall thickness.

In conclusion, relapsing polychondritis and airway involvement is a severe disease for which immunosuppressive therapy is not always sufficient for symptom improvement.
Because life-threatening complications may occur during bronchoscopic intervention, we suggest that medical therapy should always be the first-step therapy and that endobronchial intervention should be considered in subjects with insufficient improvement despite medical therapy.

Jeanne Chapron*, Delphine Wermert", Françoise Le PimpecBarthes", Aurélie Cazes ${ }^{+}$, Romain Pommier*, Anne Hernigou $^{\S}$, Jacques Lacronique*, Daniel Dusser* and Pierre-Régis Burgel $^{*}$

*Service de Pneumologie, Hôpital Cochin, AP-HP, Université Paris Descartes, Sorbonne Paris Cité, " Service de Pneumologie, Hôpital Européen Georges Pompidou, "Service de Chirurgie Thoracique, Hôpital Européen Georges Pompidou, ${ }^{+}$Service d'Anatomie-Pathologique, Hôpital Européen Georges Pompidou, and ${ }^{\S}$ Service de Radiologie, Hôpital Européen Georges Pompidou, AP-HP, Université Paris Descartes, Sorbonne Paris Cité, Paris, France.

Correspondence: P-R. Burgel, Service de Pneumologie, Hôpital Cochin, AP-HP, 27 rue du Faubourg St Jacques, 75014 Paris, France. E-mail: pierre-regis.burgel@cch.aphp.fr

Statement of Interest: D. Dusser received fees for consultancy, advisory board participation or lectures from AstraZeneca, Boehringer Ingelheim, Chiesi, Novartis and Nycomed. Industry-sponsored grants for education were received from GlaxoSmithKline and Boerhinger Ingelheim. P-R. Burgel received fees for advisory board participation or lectures from AstraZeneca, Boehringer Ingelheim, Chiesi, Novartis and Nycomed.

Provenance: Submitted article, peer reviewed.

\section{REFERENCES}

1 Lahmer T, Treiber M, von Werder A, et al. Relapsing polychondritis: an autoimmune disease with many faces. Autoimmun Rev 2010; 9: 540-546.

2 Eng J, Sabanathan S. Airway complications in relapsing polychondritis. Ann Thorac Surg 1991; 51: 686-692.

3 Ernst A, Rafeq S, Boiselle P, et al. Relapsing polychondritis and airway involvement. Chest 2009; 135: 1024-1030.

4 Dooms C, De Keukelaire T, Janssens A, et al. Performance of fully covered self-expanding metallic stents in benign airway strictures. Respiration 2009; 77: 420-426. 